\title{
Automated segmentation and recognition of the bone structure in non-contrast torso CT images using implicit anatomical knowledge
}

X. Zhou, T. Hayashi, M. Han, H. Chen, T. Hara, et al.

X. Zhou, T. Hayashi, M. Han, H. Chen, T. Hara, H. Fujita, R. Yokoyama, M. Kanematsu, H. Hoshi, "Automated segmentation and recognition of the bone structure in non-contrast torso CT images using implicit anatomical knowledge," Proc. SPIE 7259, Medical Imaging 2009: Image Processing, 72593S (27 March 2009); doi: 10.1117/12.812945

Event: SPIE Medical Imaging, 2009, Lake Buena Vista (Orlando Area), Florida, United States 


\title{
Automated segmentation and recognition of the bone structure in non-contrast torso CT images using implicit anatomical knowledge
}

\author{
X. Zhou *a, T. Hayashi ${ }^{\text {a }}$, M. Han ${ }^{\text {a }}$, H. Chen ${ }^{\text {b }}$, T. Hara ${ }^{\text {a }}$, H. Fujita ${ }^{\text {a }}$, R. Yokoyama ${ }^{\text {c }}$, \\ M. Kanematsu ${ }^{\text {c,d }}$, and H. Hoshi ${ }^{\text {e }}$ \\ ${ }^{a}$ Department of Intelligent Image Information, Division of Regeneration and Advanced Medical \\ Sciences, Graduate School of Medicine, Gifu University, Gifu, Japan \\ ${ }^{b}$ Department of Anatomy, Division of Disease Control, Graduate School of Medicine, Gifu \\ University, Gifu, Japan \\ ${ }^{c}$ Radiology Services, Gifu University Hospital, Gifu, Japan \\ ${ }^{\mathrm{d}}$ Department of Radiology, Gifu University Hospital, Gifu, Japan \\ e: Department of Radiology, Division of Tumor Control, Graduate School of Medicine, Gifu \\ University, Gifu, Japan
}

\begin{abstract}
X-ray CT images have been widely used in clinical diagnosis in recent years. A modern CT scanner can generate about $1000 \mathrm{CT}$ slices to show the details of all the human organs within 30 seconds. However, CT image interpretations (viewing 500-1000 slices of CT images manually in front of a screen or films for each patient) require a lot of time and energy. Therefore, computer-aided diagnosis (CAD) systems that can support CT image interpretations are strongly anticipated. Automated recognition of the anatomical structures in CT images is a basic pre-processing of the CAD system. The bone structure is a part of anatomical structures and very useful to act as the landmarks for predictions of the other different organ positions. However, the automated recognition of the bone structure is still a challenging issue. This research proposes an automated scheme for segmenting the bone regions and recognizing the bone structure in noncontrast torso CT images. The proposed scheme was applied to 48 torso CT cases and a subjective evaluation for the experimental results was carried out by an anatomical expert following the anatomical definition. The experimental results showed that the bone structure in $90 \%$ CT cases have been recognized correctly. For quantitative evaluation, automated recognition results were compared to manual inputs of bones of lower limb created by an anatomical expert on 10 randomly selected CT cases. The error (maximum distance in 3D) between the recognition results and manual inputs distributed from 3-8 $\mathrm{mm}$ in different parts of the bone regions.
\end{abstract}

Keywords: Recognition, bone structures, torso CT images, anatomical knowledge.

\section{INTRODUCTION}

The remarkable progress in multi-slice X-ray CT tomography enables the acquisition of the whole human torso in as little as 20-30 seconds. The volumetric torso CT scan contains details of all anatomical structures in the torso region. This enables the detection of lesions in different organs and tissue regions in the human torso. On the other hand, interpretation of a torso CT case (800-1000 CT slices) in front of a screen without any overlook of the lesion information needs a lot of time and experience; therefore the computer-aided diagnosis (CAD) systems are expected to reduce the burden and increase the accuracy of the interpretations by radiologists.

During the development of the CAD system, segmenting the different organ and tissue regions and recognizing the anatomical structures in CT images are basic tasks and should be solved firstly [1]. In torso CT images, bone structure is a part of anatomical structures and very useful to act as the landmarks for predictions of the other different organ positions. Although the segmentation of the main part of the bone regions in CT images is not difficult[2], recognizing the bone structures automatically in whole torso regions based on the anatomical definition is still a challenging issue and has not been resolved[3,4].

*zxr@fjt.info.gifu-u.ac.jp; phone 81-58-230-6510; fax 81-58-230-6514; Gifu University

Medical Imaging 2009: Image Processing, edited by Josien P. W. Pluim, Benoit M. Dawant, Proc. of SPIE Vol. 7259, 72593S - ( 2009 SPIE

CCC code: $1605-7422 / 09 / \$ 18 \cdot$ doi: $10.1117 / 12.812945$

Proc. of SPIE Vol. 7259 72593S-1 
In this paper, we propose a fully automated scheme to segment the 3-D bone regions in non-contrast torso CT images using a density based method and divide the bone regions into different parts using implicit anatomical knowledge. This research work is a part of our research that aimed to recognize the whole anatomical structures of human torso using high-resolution CT images.

\section{METHODS}

This scheme includes two principal parts: bone region segmentation and bone structure recognition as shown in Fig. 1. Both parts are designed as fully automatic processes based on non-contrast torso CT images.

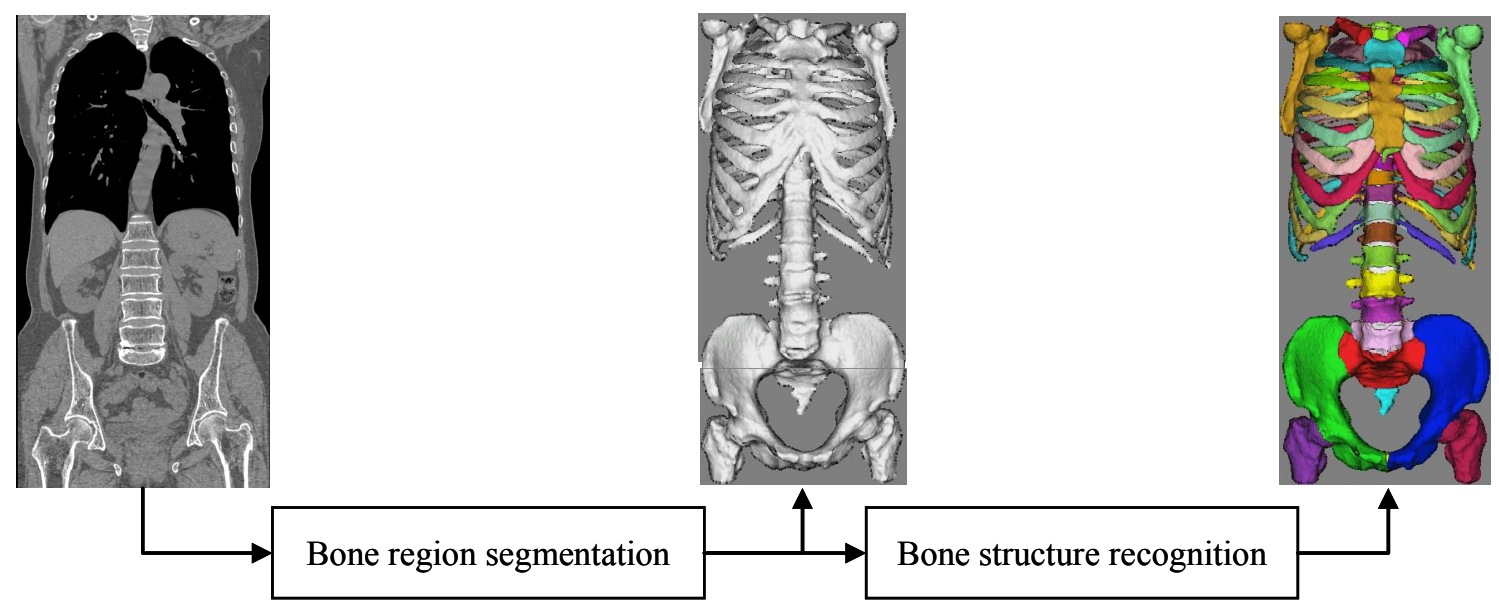

Fig. 1 Outline of the scheme for bone structure recognition.

\subsection{Bone region segmentation using torso $\mathrm{CT}$ images}

The CT numbers of bone regions are higher than the other tissue regions of human body in non-contrast CT images. So that, the density (CT number) based methods such as gray-level thresholding or region growing are effective for segmenting the bone regions in CT images. We use the simplest method (a gray-level thresholding) to keep the high generality and capacity of the segmentation process and optimize its parameter (a threshold value) for each patient specifically to get the high accuracy during the segmentation of the bone regions in CT images. Even the most part of the bone regions have the high $\mathrm{CT}$ numbers that can be distinguished from the other tissues, the CT number distribution of the cartilage regions overlapped with the CT number distributions of liver and muscle regions in the histogram of the CT images. It is difficult to find an optimum threshold value that can separate the cartilage with other tissues properly. In order to solve this problem, we propose a new method to decide the optimum threshold value for each patient case specifically using a dynamic histogram analysis. This analysis assumes the bone region and liver region as Gaussian distributions and searches the best separation point (threshold value) of those two distributions by observing its variations. The gray level thresholding with an optimum threshold value decision is an effective and fast solution for the bone segmentation.

\subsection{Bone structure recognition}

After the segmentation of the bone regions, the bone regions are divided and categorized into the different parts based on the anatomical definition. The processing flow is shown in the Fig. 2. The bone regions are divided and categorized into 60 categories such as vertebras (18), ribs (12), sternum (3) and so on. The approach recognizes each part sequentially based on implicit anatomical knowledge through 8 main steps as shown in Fig.2. The bone regions are modeled as a connection of 60 components firstly, and then a coarse-to-fine, global-to-local bone region splitting is proceeded to accomplish the bone structure recognition. The patterns of the connection, shape, and spatial relationships are used as the features during the recognition. 


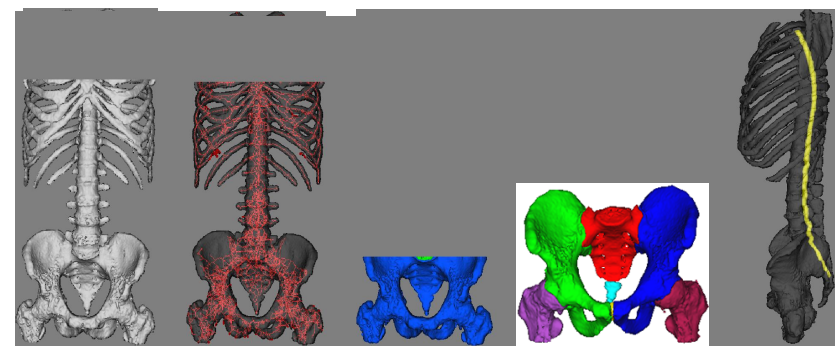

(a)

(b)

(c)

(d)

(e)

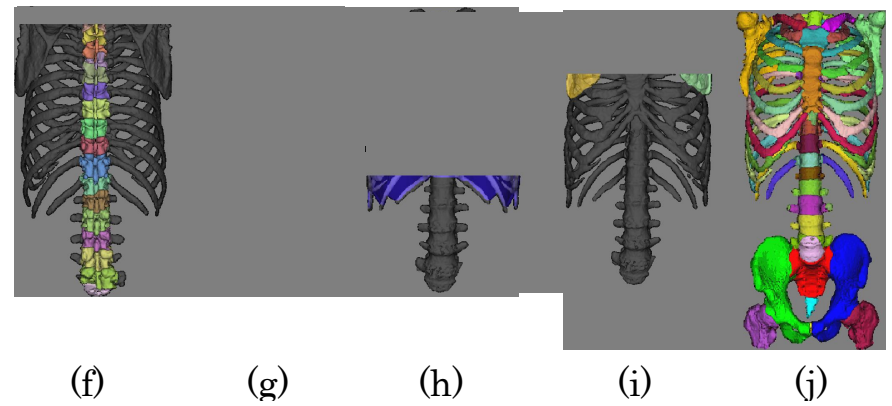

Fig. 2 An example of recognition results of the bone structures in torso CT images.

(a) bone regions in torso CT images, (b) axial lines of the bone regions, (c) identification of lower limb,(d) structure of lower limb, (e) vertebral canal, (f) recognition results of vertebras, (g) sternum and ribs, (h) thoracic cavity, (i) scapula and clavicle, (j) final result. (Different parts in the bone structures were showed by different colors).

\section{EXPERIMENTS AND RESULTS}

The proposed scheme was applied to 48 cases (male: 38; female: 10; ages: 20-88) of non-contrast CT images. Each CT image covers the whole torso region with an isotopic spatial resolution of about $0.63 \mathrm{~mm}$ and a 12 bits density resolution. A recognition result of the bone structure with the original CT images was shown in Fig.3(c). The manual input result of the bone structures (Fig.3(b)) were used as the ground truth for accuracy evaluation.

We confirmed the bone structure in $90 \%$ CT cases was recognized successfully based on a subjective validation by an anatomical expert. The successful ratios of vertebras, ribs, sternum and bones of upper limb recognitions were $92 \%, 98 \%$, $90 \%$ and $96 \%$ respectively. A quantitative evaluation based on ground truths was carried out for bones of lower limb. The ground truths of bones of lower limb that included femur, sacrum, hip bone, coccyx were manually generated by an anatomical expert on randomly selected $10 \mathrm{CT}$ cases. The maximum values of 3-D distances (MaxD) between recognition results and ground truths of thigh bone, sacrum, hip bone and coccyx were measured. The mean values (standard deviations) of MaxDs on femur, sacrum, hip bone and coccyx were $3.27 \mathrm{~mm}$ (2.79), $4.58 \mathrm{~mm}(1.24), 7.13 \mathrm{~mm}$ (1.47) and $1.76 \mathrm{~mm}(0.57)$ respectively.

\section{CONCLUSION}

We developed a fully automated scheme to segment the 3-D bone regions in non-contrast torso CT images and recognize the bone structure. We applied this scheme to 48 torso CT scans and confirmed that the bone region in all the CT cases was segmented almost correctly and bone structure was recognized successfully in $90 \%$ CT cases comparing with the general definition of human anatomy. The recognition results should be useful for computer-aided diagnosis of 
the bone diseases (e.g. osteoporosis) and provide the spatial reference for identifying the other organ positions in CT images.

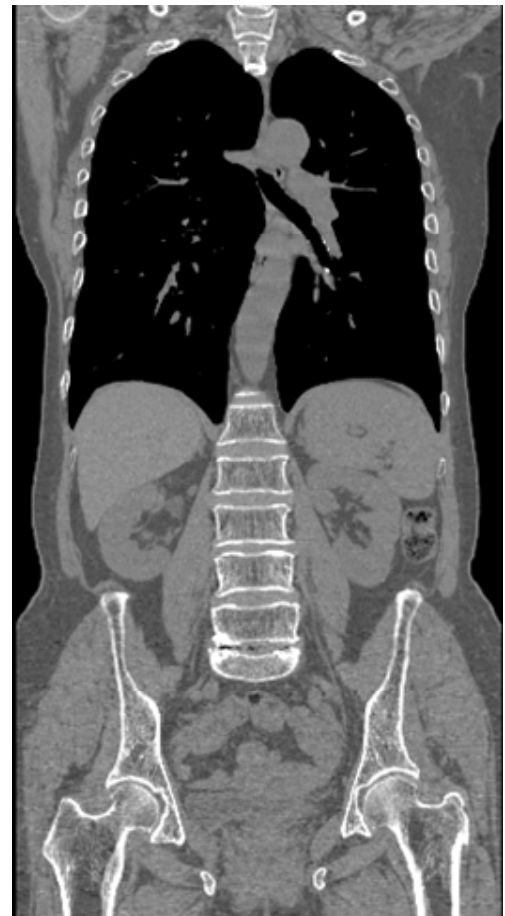

(a) CT images (1 coronal slice )

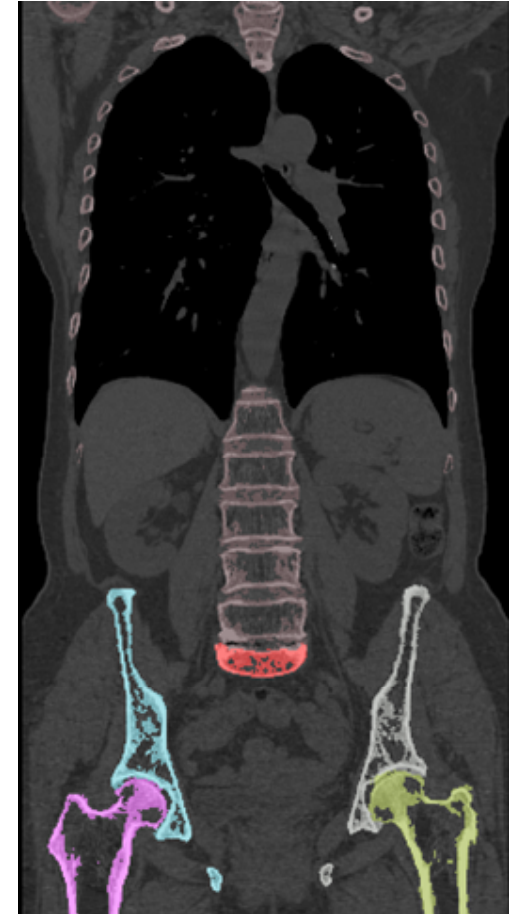

(b) Manual input of the bone structure

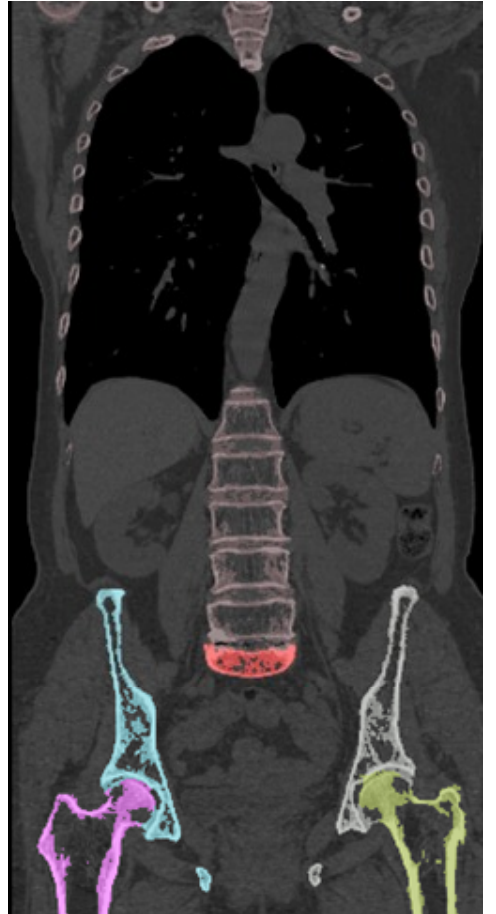

(c) Recognition result

Fig. 3 Recognition results (right) and ground truth (middle) of bone structure

(Different parts in bone structures were showed by the different colors).

\section{ACKNOWLEDGEMENTS}

Authors thank members of the Fujita Laboratory for their valuable discussions. This research was supported in part by a research grant from Kayamori Foundation of Informational Science Advancement, in part by a research grant from Gifu University, and in part by Ministry of Health, Labour, and Welfare under a Grant-In-Aid for Cancer Research, Japanese Government.

\section{REFERENCES}

1. X. Zhou, T. Hara, H. Fujita, R. Yokoyama, T. Kiryu, M. Kanematsu, and H. Hoshi: Preliminary study for automated recognition of anatomical structure from torso CT images, Proc. of the 2005 IEEE Engineering in Medicine and Biology 27th Annual Conference, paper\#340, 2005.

2. X. Zhou, T. Hara, H. Fujita, R. Yokoyama, T. Kiryu, and H. Hoshi: Automated segmentations of skin, soft-tissue, and skeleton, from torso CT images. Proc. SPIE Medical Imaging 2004, 5370: 1634-1639, 2004.

3. X. Zhou, S. Kobayashi, T. Hara, H. Fujita, R. Yokoyama, T. Kiryu, and H. Hoshi: Atlas-based structure recognition of chest bone frames from torso multi-slice CT images. Medical Imaging and Information Sciences, 22 (3): 220-228 2005 (In Japanese).

4. X. Zhou, S. Kobayashi, T. Hara, H. Fujita, R. Yokoyama, T. Kiryu, and H. Hoshi: Extraction of thoracic cage based on chest bone frame information from 3-D torso multi-slice CT images. IEICE D-II, J88-D-II (9): 1999-2002: 2005 (In Japanese). 